\title{
PADRONIZAÇÃO DA MULTIPLE SCLEROSIS FUNCTIONAL COMPOSITE MEASURE (MSFC) NA POPULAÇÃO BRASILEIRA
}

\author{
Charles P. Tilbery ${ }^{1}$, Maria Fernanda Mendes², Rodrigo Barbosa Thomaz ${ }^{3}$, \\ Bianca Etelvina Santos de Oliveira ${ }^{4}$, Giorge Luiz Ribeiro Kelian ${ }^{5}$, \\ Roberta Busch ${ }^{4}$, Patrícia Príncipe Carvalho Miranda ${ }^{4}$, Paula Caleffi ${ }^{4}$
}

\begin{abstract}
RESUMO - A Multiple Sclerosis Functional Composite Measure (MSFC) é escala para avaliação dos pacientes portadores de esclerose múltipla, desenvolvida pela National Multiple Sclerosis Society dos EUA em 1994, que envolve uma composição de três testes - 9-Hole Peg Test, Timed 25-Foot Walk e PASAT - abrangendo de maneira multidimensional as principais funções neurológicas comprometidas nestes pacientes. A MSFC foi aplicada em 91 indivíduos sadios com o objetivo de padronizá-la na população brasileira para posterior uso nos diversos centros de tratamento e pesquisa no Brasil.
\end{abstract}

PALAVRAS-CHAVE: esclerose múltipla, MSFC, padronização, Brasil.

\begin{abstract}
Multiple Sclerosis Functional Composite Measure (MSFC) standardized in Brazilian population
ABSTRACT - The Multiple Sclerosis Functional Composite Measure (MSFC) is an outcome measure in multiple sclerosis developed by USA National Multiple Sclerosis Society (1994), a three-part composite clinical measure - 9-Hole Peg Test, Timed 25-Foot Walk and PASAT. It should be multidimensional in order to reflect the principal ways MS affects an individual. The MSFC was applied in 91 Brazilian subjects and standardized to be use in MS centers.
\end{abstract}

KEY WORDS: multiple sclerosis, MSFC, standardize, Brazil.

As manifestações clínicas da esclerose múltipla (EM) são variáveis de paciente para paciente e sua evolução usualmente não é linear, caracterizada por piora dos sintomas, acúmulo de incapacidades intercalados, no início da doença, por períodos variáveis de melhora. Estas variações são difíceis de quantificar na prática neurológica, motivo pelo qual os instrumentos disponíveis para estas avaliações terem sido até a presente data um desafio constante ${ }^{1,2}$.

A Sociedade Americana de Esclerose Múltipla desenvolveu em 1994 nova escala de avaliação de pacientes com EM, a Multiple Sclerosis Functional Composite Measure (MSFC), instrumento atualmente utilizado de maneira crescente por centros de tratamento de pacientes com a doença ${ }^{1,3}$.

Com a finalidade de aplicar este teste nos portadores de EM no Brasil, a MSFC foi aplicada em indivíduos sadios com intuito de padronizá-lo na população brasileira.

\section{MÉTODO}

Sujeitos - Foram avaliados 91 voluntários normais, recrutados entre familiares e amigos dos pacientes que freqüentam o Centro de Atendimento e Tratamento de Esclerose Múltipla da Santa Casa de São Paulo (CATEM). Para obedecer aos critérios necessários para validação para a população brasileira, as variáveis de sexo, idade e escolaridade foram controladas no momento de inclusão dos sujeitos na amostra. Avaliamos 49 indivíduos do feminino e 42 do masculino, com faixa etária variando entre 20 e 50 anos e a escolaridade entre 4 e 15 anos (ou mais). Foram criadas três faixas de idade (20-29 anos, 30-39 anos e 40-50 anos) e de escolaridade (4 a 8 anos, 9 a 14 anos, 15 anos ou mais). Com distribuição homogênea dos sujeitos entre os grupos quanto as variáveis pré-estabelecidas.

Esta pesquisa foi submetida à aprovação do Comitê de Ética da Santa Casa de São Paulo.

Material - Para a elaboração da MSFC, utilizamos o material doado pelo Laboratório Abbott do Brasil Ltda,

Centro de Atendimento e Tratamento da Esclerose Múltipla (CATEM) - Disciplina de Neurologia da Faculdade de Ciências Médicas

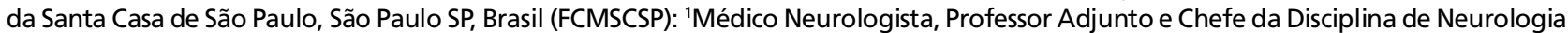
da Santa Casa de São Paulo; ${ }^{2}$ Médica Neurologista, Professora Assistente da Disciplina de Neurologia da FCMSCSP; ${ }^{3}$ Médico Neurologista, Pós-graduando do CATEM; ${ }^{4}$ Fonoaudiólogas da Santa Casa de São Paulo. 
composto por: 1) Manual de Administração e Escores para o MSFC, 2) Kit para a realização do 9-HPT, 3) Fita Cassete, com gravação completa do PASAT, em inglês. Os testes foram traduzidos e/ou adaptados de acordo com a sua necessidade, conforme descreveremos a seguir, sendo seguidas as instruções do manual ${ }^{4}$.

Para a realização do Teste Caminhada Cronometrada de 25 pés (Timed 25-Foot Walk - 25-FW), foi pintada uma faixa branca, com 7,62 metros em um corredor do Hospital Central da Santa Casa de São Paulo, coberto, com piso antiderrapante. $O$ sujeito da pesquisa era orientado a utilizar sapatos confortáveis, não sendo permitido ca rregar nenhum objeto durante a prova. Um cronômetro é necessário para a medição do tempo, que é expresso em décimos de segundo (Ex. 35,45" deverá ser arredondado para 35,5" e 35,44" para 35,4"). São realizadas duas tentativas consecutivas, sendo o tempo máximo de 180 segundos por tentativa.

No Teste dos 9 Pinos nos Buracos (9-Hole Peg Test 9-HTP), é necessário o equipamento adequado, apoiado sobre uma mesa, com o sujeito confortavelmente sentado. A plataforma de testes é composta por nove orifícios, um compartimento para os pinos, com um total de 15 pinos. O equipamento é disposto horizontalmente em frente ao sujeito, de forma que o compartimento que contém os pinos fique voltado para a mão que será testada. $O$ teste será realizado duas vezes em cada mão, sendo duas tentativas consecutivas com a mão dominante, seguidas imediatamente por duas tentativas consecutivas com a mão não dominante. A mão utilizada com maior freqüência para a escrita foi considerada como a mão dominante. Um cronômetro foi utilizado para a medição do tempo, expresso em décimos de segundos. $O$ tempo máximo permitido por teste é 300 segundos.

A versão brasileira do Teste Auditivo Compassado de Adição Seriada (Paced Auditory Serial Addition Task PASAT) foi elaborada, gravada e aplicada por uma equipe de fonoaudiólogas. Os estímulos foram gravados em cabine acústica através de sistema digital, nas duas versões, com intervalos interestímulos de três e dois segundos. Para evitar distorções após utilizações repetidas, utilizamos a gravação em Compact Disc. Foi realizada a gravação de três seqüências de treinamento com 11 estímulos cada, para o teste de treinamento de $3 "$ e de $2 "$. A forma A e B do teste foram gravadas com 3" e 2", sendo que cada uma delas contém 61 estímulos. Neste teste, os estímulos são algarismos de um a nove, apresentados em seqüência aleatória e pré-determinada. A tarefa consiste em realizar a soma dos números apresentados, dois a dois, desconsiderando o resultado do cálculo, assim, se a seqüência for "7, 8, 6, 3 e 7" a resposta correta será "15, 14, 9 e 10". O teste é iniciado com a apresentação da seqüência numérica a cada 3". Para a aplicação do teste, é necessário ambiente silencioso, sem estímulos visuais ou auditivos, apenas com a presença do sujeito e do examinador na sala. $O$ escore será determinado pelo número de respostas corretas, sendo que o escore máximo em cada parte é 60 pontos.
Para o 9-HPT e para o PASAT, utilizamos sala com isolamento acústico, estando presentes apenas o examinador e o sujeito.

Procedimentos - Os componentes da MSFC deverão ser aplicados na seguinte ordem: $1^{\circ}$ ) $25-\mathrm{FW}$ - tentativa 1 e $2 ; 2^{\circ}$ ) 9-HPT - mão dominante - tentativa 1 e $2 ; 3^{\circ}$ ) 9 HPT - mão não dominante - tentativa 1 e $2 ; 4^{\circ}$ ) PASAT 3 "e $5^{\circ}$ ) PASAT 2".

Instruções para Teste da Caminhada Cronometrada (25-FW) - O sujeito é encaminhado a uma das extremidades marcadas para a prova, e orientado para aguardar a ordem para iniciar, sendo dada a seguinte instrução: "Eu gostaria que você andasse 7,62m o mais rápido que puder. Não diminua o passo até passar a linha de chega da. Pronto? Agora!". O cronômetro é acionado quando o pé cruza a linha de partida e desligado quando o pé atravessa a linha de chegada. Para a segunda tentativa, o sujeito é encaminhado para a linha de partida e as instruções são repetidas.

Instruções para o Teste dos 9 Pinos nos Buracos (9-HPT) - Após o sujeito estar acomodado, a seção é iniciada com o examinador perguntando "Agora iremos avaliar a sua habilidade com as mãos e os braços. Você utiliza a mão direita ou a esquerda para escrever?". O equipamento deverá ser adequadamente posicionado e as seguintes instruções deverão ser lidas para o paciente: "Neste teste, você deverá pegar estes pinos e coloca-los nos buracos o mais rápido que puder, até que todos os buracos estejam pre enchidos. A seguir, sem parar, retire os pinos dos buracos e os coloque novamente no coletor. Durante toda a prova, você deverá pegar um pino por vez, e utilizar somente a mão que estiver sendo testada. Se algum pino cair no chão, ignore-o e continue o teste, que eu o pegarei para você. Este teste será feito duas vezes com cada mão. Nós inicia remos com a sua mão [dominante]. Se você quiser segurar a prancha, use a mão que não está sendo testada. Você tem alguma dúvida? Então, vamos ver o quão rápido você consegue colocar e retirar os pinos dos buracos. Pronto? Comece!". O cronômetro deverá ser acionado quando o sujeito tocar no primeiro pino, e desligado quando o último for colocado no coletor. Após a primeira tentativa com a mão dominante (MD), o examinador diz: "Ótimo! Agora eu gostaria que você repetisse a prova mais uma vez com a sua mão [dominante]. Veja o quão rápido consegue co locar e retirar os pinos. Pronto? Comece!".

Após a segunda tentativa com a MD, o equipamento deverá ser colocado de forma que o compartimento que contém os pinos fique na frente da mão não dominante (MND). Então diga: "Muito bem, agora eu gostaria que você realizasse a prova com a sua mão [não dominan te]. Desta vez, utilize a mão [dominante] para estabilizar a prancha. Pronto? Comece!". O procedimento deverá ser realizado da mesma maneira que com a MD, nas duas tentativas. Os tempos deverão ser anotados após cada tentativa. 
Instruções para o PASAT - Antes de iniciar o teste, deverá ser apresentada a versão de treinamento com 10 itens. Ela deverá ser aplicada no mínimo uma vez e no máximo três vezes. A aplicação do PASAT terá início com o treinamento da parte 1, com estímulos a cada 3", e é dado o seguinte comando: "Você ouvirá uma série de números que serão apresentados um a um, a cada três segundos. Ouça os dois primeiros números, some-os e diga-me a resposta. Quando você ouvir o próximo, someo com aquele que você ouviu imediatamente antes de realizar a soma. Continue sempre somando os dois últi mos números que você ouvir. Lembre-se, eu não estou pedindo a soma total de todos os números e sim a soma dos dois últimos números que são falados na gravação. Por exemplo, se os dois primeiros números forem " 5 " e "7", a resposta correta seria "12". Se o próximo número for "3", a resposta será "10" e se o próximo for "2", a res posta será "5". Você entendeu?". Caso as dúvidas persistam, o exemplo deverá ser repetido com uma demonstração escrita de como o teste deverá ser realizado. Então prossiga: "Esta é uma tarefa desafiadora. Se você se per der, não se preocupe, apenas pule a resposta, escute os próximos dois números, some-os e continue o teste. Va mos treinar um pouco antes de começar?".

Durante o período de treinamento, o teste poderá ser interrompido se ficar claro que o sujeito não compreendeu. Caso apenas cometa erros, prossiga com a aplicação. Se o sujeito não responder por duas vezes consecutivas, oriente: "Escute os próximos dois números e continue". Ao término da fase de treinamento, o teste deverá ser iniciado. Antes de iniciar o PASAT 3", lembre ao paciente: "Lembre-se, assim que iniciarmos o teste, não é mais possível interrompe-lo. Se você estiver perdido, simplesmente preste atenção nos próximos dois números. Ainda tem alguma dúvida? Então, vamos começar". Durante a aplicação do teste, caso o paciente deixe de responder cinco respostas consecutivas, redirecione-o rapidamente, dizendo "Pule para a próxima", mas não pare a fita nem interrompa o teste.

A segunda parte (estímulos a cada 2") deverá ser iniciada imediatamente após o término da parte 1 , sem período de descanso. Diga para o sujeito: "Esta segunda parte do teste é idêntica a anterior. A única diferença é que os números serão ditos mais rapidamente, a cada dois segundos. Vamos treinar pelo menos uma vez?". É obrigatório realizar pelo menos um treino antes de iniciar a Parte 2. Inicie a aplicação imediatamente após o treinamento, seguindo as mesmas instruções do PASAT 3". Para a correção do teste, o examinador deverá marcar com um círculo as respostas corretas, anotar as respostas erradas ou NR (não respondeu) quando nenhuma resposta for dada. Caso o sujeito corrija a resposta, esta deverá ser aceita e considerada no escore final. Anote se houver auto-correção (AC). Os escores correspondem ao número de respostas corretas em cada uma das versões.
Composição do escore da MSFC - São utilizados três componentes para a determinação da MSFC: (1) o escore médio das duas tentativas do 25-FW; (2) o escore médio das quatro tentativas do 9-HPT, sendo considerada a média recíproca entre as duas tentativas com a MD e com a mão MND; (3) o número de respostas corretas no PASAT 3". Para a criação de um escore-Z baseado numa população normal como referência, utilizamos as seguintes fórmulas:

a) $Z$ pernas $=$ [média sujeito (25-FW) - média do grupo (25-FW) /desvio padrão do grupo (25-FW)]

b) $Z$ braços = [média sujeito (1/9-HPT) - média do grupo (1/9-HPT) /desvio padrão do grupo (1/9-HPT)]

c) $Z$ cognitivo = [média sujeito (PASAT-3") - média do grupo (PASAT-3") /desvio padrão do grupo (PASAT-3")]

Após determinarmos as médias e os desvios padrões de referência para a população brasileira, será criado um escore para a MSFC que tenha como base indivíduos sadios da população brasileira, obedecendo a seguinte fórmula:

MSFC escore $=(Z$ braços $-Z$ pernas $+Z$ cognitivo $) / 3$

Método estatístico - Aplicamos o teste t-Student, controlado pelo teste de Levene para a igualdade de variâncias, com a intenção de observarmos possíveis diferenças entre os gêneros, para os três componentes da MSFC. As possíveis diferenças entre as faixas etárias e escolaridade foram estudadas pela análise das variâncias (ANOVA). Quando este apresentava diferenças estatisticamente significantes, os testes de Turkey e/ou Dunnett foram aplicados posteriormente para identificarmos as diferenças entre as médias. Quando $p<5 \%(0,05)$ consideramos haver diferença estatisticamente significante.

\section{RESULTADOS}

Os resultados dos testes estão demonstrados nas Tabelas 1, 2 e 3.

Após a análise dos dados a fórmula final para ser usada na população brasileira é a seguinte:

MSFC escore $=\{($ Média sujeito $[1 / 9-\mathrm{HPT}]$ 0,0508/0,0058) - (Média sujeito [25-FW] - 4,09/0,46) + (PASAT 3" - 39,71/13,23)\}/3

\section{DISCUSSÃO}

A avaliação longitudinal do paciente com EM é realizada principalmente com a Expanded Disability Status Scale - EDSS 5 que, por sua familiaridade, abrangência das várias funções do sistema nervoso e facilidade de aplicação tem sido até a presente data a mais utilizada ${ }^{6-9}$. A escala EDSS tem grande influência da capacidade de deambulação nos escores intermediários, tornando-se insensível a alterações de outras funções neurológicas principalmente em pa- 
Tabela 1. Demonstração dos resultados do "Teste Caminhada Cronometrada de 25 pés " (Timed 25-Foot Walk) aplicado em 91 sujeitos sadios, medido em segundos, de acordo com sexo, escolaridade e faixa etária.

Caminhada cronometrada Sexo de 25 pés

\begin{tabular}{|c|c|c|c|c|c|c|c|c|c|c|c|}
\hline & $\mathrm{F}$ & $M$ & $\mathrm{p}$ & $\begin{array}{c}4-8 \\
\text { anos }\end{array}$ & $\begin{array}{l}9-14 \\
\text { anos }\end{array}$ & $\begin{array}{l}\geq 15 \\
\text { anos }\end{array}$ & $\mathrm{p}$ & $\begin{array}{c}20-29 \\
\text { anos }\end{array}$ & $\begin{array}{c}30-39 \\
\text { anos }\end{array}$ & $\begin{array}{r}40-50 \\
\text { anos }\end{array}$ & $p$ \\
\hline$n$ & 49 & 42 & & 30 & 30 & 31 & & 30 & 30 & 31 & \\
\hline \multicolumn{12}{|l|}{$1^{\circ}$ teste } \\
\hline média (seg) & 4,27 & 3,99 & 0,006 & 4,19 & 4,13 & 4,11 & 0,781 & 4,09 & 4,13 & 4,22 & 0,561 \\
\hline $\mathrm{dp}$ & $(0,43)$ & 0,50 & * & 0,44 & 0,55 & 0,46 & & 0,58 & 0,44 & 0,43 & \\
\hline \multicolumn{12}{|l|}{$2^{\circ}$ teste } \\
\hline média (seg) & 4,17 & 3,88 & 0,001 & 4,05 & 3,99, & 4,07 & 0,790 & 3,96 & 4,05 & 4,10 & 0,436 \\
\hline $\mathrm{dp}$ & 0,42 & 0,41 & * & 0,39 & 0,51 & 0,42 & & 0,52 & 0,37 & 0,41 & \\
\hline
\end{tabular}

F, feminino; M, masculino; seg, segundos; dp, desvio padrão. $p>5 \%(0,05)$ estatisticamente significante. * estatisticamente significante para o teste t-Student.

Tabela 2. Demonstração dos resultados do "Teste do 9 Pinos nos Buracos" (9-Hole Peg Test) aplicado em 91 sujeitos sadios, medido em segundos, de acordo com sexo, escolaridade e faixa etária.

\begin{tabular}{|c|c|c|c|c|c|c|c|c|c|c|c|}
\hline & \multicolumn{2}{|c|}{ Sexo } & \multirow[b]{2}{*}{$\mathrm{p}$} & \multicolumn{3}{|c|}{ Escolaridade } & \multicolumn{5}{|c|}{ Faixas Etárias } \\
\hline & $\mathrm{F}$ & $M$ & & $\begin{array}{c}4-8 \\
\text { anos }\end{array}$ & $\begin{array}{l}9-14 \\
\text { anos }\end{array}$ & $\begin{array}{l}\geq 15 \\
\text { anos }\end{array}$ & $p$ & $\begin{array}{c}20-29 \\
\text { anos }\end{array}$ & $\begin{array}{r}30-39 \\
\text { anos }\end{array}$ & $\begin{array}{r}40-50 \\
\text { anos }\end{array}$ & $\mathrm{p}$ \\
\hline $\mathrm{n}$ & 49 & 42 & & 30 & 30 & 31 & & 30 & 30 & 31 & \\
\hline \multicolumn{12}{|l|}{ mão } \\
\hline \multicolumn{12}{|l|}{ dominante } \\
\hline \multicolumn{12}{|l|}{$1^{\circ}$ teste } \\
\hline média (seg) & 18,9 & 20,3 & 0,013 & 20,4 & 19,2 & 19,0 & 0,091 & 18,07 & 19,5 & 20,4 & 0,059 \\
\hline $\mathrm{dp}$ & 2,4 & 3,0 & * & 2,8 & 2,6 & 2,7 & $* *$ & 2,5 & 2,6 & 3,0 & $* * * *$ \\
\hline \multicolumn{12}{|l|}{$2^{\circ}$ teste } \\
\hline média (seg) & 17,7 & 18,7 & 0,093 & 18,9 & 17,6 & 17,9 & 0,152 & 17,7 & 17,7 & 19,0 & 0,099 \\
\hline $\mathrm{dp}$ & 2,7 & 2,7 & & 3,5 & 1,8 & 2,4 & & 2,6 & 2,1 & 3,3 & $* * * *$ \\
\hline \multirow{2}{*}{\multicolumn{12}{|c|}{$\begin{array}{l}\text { mão não } \\
\text { dominante }\end{array}$}} \\
\hline & & & & & & & & & & & \\
\hline \multicolumn{12}{|l|}{$1^{\circ}$ teste } \\
\hline média (seg) & 20,2 & 20,6 & 0,525 & 21,5 & 20,0 & 19,7 & 0,085 & 19,3 & 20,6 & 21,4 & 0,026 \\
\hline $\mathrm{dp}$ & 3,3 & 2,8 & & 4,3 & 2,3 & 1.7 & $* *$ & 1,9 & 2,6 & 4,1 & $* * *$ \\
\hline \multicolumn{12}{|l|}{$2^{\circ}$ teste } \\
\hline média (seg) & 19,9 & 20,2 & 0,615 & 21,6 & 18,9 & 19,5 & 0,062 & 19,2 & 19,6 & 21,3 & 0,092 \\
\hline $\mathrm{dp}$ & 3,2 & 3,5 & & 4,5 & 1,9 & 2,1 & ** & 2,4 & 2,1 & 4,6 & $* * * *$ \\
\hline
\end{tabular}

F, feminino; $M$, masculino; seg, segundos; dp, desvio padrão; $p>5 \%(0,05)$ estatisticamente significante; * estatisticamente significante para o sexo feminino pelo teste t-Student; **tendência à significância estatística entre $1^{\circ}$ e $3^{\circ} \mathrm{grau}$ pelo teste de Dunnett; ***estatisticamente significante para a maior faixa etária pelo teste de Tukey; ${ }^{* * *}$ tendência à significância estatística para a maior faixa etária em relação à menor, pelos testes de ANOVA e Tukey.

cientes com incapacidades moderadas e/ou graves, ignorando disfunções cognitivas freqüentes neste pacientes. Estas ambigüidades contribuem para significante variabilidade entre vários examinadores ${ }^{10-12}$.

Escalas alternativas foram desenvolvidas ${ }^{13-14}$, e ntretanto apresentam as mesmas limitações psicométricas.
Os princípios que nortearam a criação da MSFC incluem medidas que refletem as dimensões das alterações clínicas dos pacientes com mais fidelidade, evitam redundância nas medidas, utilizam instrumentos menos complexos e finalmente, enfatizam alterações com maior sensibilidade ${ }^{1,15}$. A MSFC correlacionou-se com os componentes individuais 
Tabela 3. Demonstração dos resultados do "Teste Auditivo Compassado de Adição Seriada " (Paced Auditory Serial Addition Task - PASAT) aplicado em 91 sujeitos sadios, avaliado através de pontuação, de acordo com sexo, escolaridade e faixa etária.

Teste auditivo

compassado

Sexo

Escolaridade

Faixas Etárias

de adição

seriada

\begin{tabular}{|c|c|c|c|c|c|c|c|c|c|c|c|}
\hline & $\mathrm{F}$ & M & $p$ & $\begin{array}{c}4-8 \\
\text { anos }\end{array}$ & $\begin{array}{l}9-14 \\
\text { anos }\end{array}$ & $\begin{array}{l}\geq 15 \\
\text { anos }\end{array}$ & $p$ & $\begin{array}{c}20-29 \\
\text { anos }\end{array}$ & $\begin{array}{l}30-39 \\
\text { anos }\end{array}$ & $\begin{array}{l}40-50 \\
\text { anos }\end{array}$ & $p$ \\
\hline $\mathrm{n}$ & 49 & 42 & & 30 & 30 & 31 & & 30 & 30 & 31 & \\
\hline \multicolumn{12}{|l|}{$1^{\circ}$ teste -3 seg } \\
\hline média (pontos) & 37,6 & 42,1 & 0,105 & 36,2 & 39,4 & 43,6 & 0,088 & 40,1 & 37,5 & 41,8 & 0,451 \\
\hline$d p$ & 13,3 & 12,9 & & 13,0 & 13,3 & 12,8 & $* *$ & 14,3 & 14,0 & 11,1 & \\
\hline \multicolumn{12}{|l|}{$2^{\circ}$ teste -2 seg } \\
\hline média (pontos) & 29,2 & 36,1 & 0,007 & 28,7 & 31,3 & 37,5 & 0,017 & 34,6 & 30,2 & 32,5 & 0,393 \\
\hline $\mathrm{dp}$ & 11,6 & 12,3 & * & 12,5 & 10,7 & 12,6 & $\star * *$ & 14,0 & 13,5 & 8,7 & \\
\hline
\end{tabular}

F, feminino; $M$, masculino; seg, segundos; dp, desvio padrão; $p>5 \%(0,05)$ estatisticamente significativo; *estatisticamente significante pelo teste $t-$ Student; ** diferença estatisticamente significante para escolaridade, entre $1^{\circ}$ e $3^{\circ}$ grau, pelo teste de Tukey. Obs: entre $1^{\circ}$ e $2^{\circ}$ grau, $p=0,662 ;$ entre $2^{\circ}$ e $3^{\circ}$ grau, $p=0,138$.

do EDSS, porém com informações adicionais mais explícitas e com alterações das funções cognitivas em trabalhos anteriores ${ }^{2}$. Estas escalas não se correlacionam com achados nos exames de ressonância magnética ${ }^{16}$.

Uma das características da MSFC é quantificar o comprometimento neurológico relativo combinando os resultados dos três testes 9-HTP, 25-FW e PASAT, que são expressos em unidades diferentes (segundos e números de acertos) em um escore único, que padronizados, criam um escore final, chamado escore-Z. Este escore-Z indica quanto (unidades ou desvios-padrão) o teste resulta em desvio de uma população utilizada com padrão, de tal maneira que utilizando populações distintas os escores-Z deverão variar caso a caso. Considerando este fato, resolvemos verificar o escore- $Z$ esperado para a população brasileira, visto haver diferenças étnicas envolvendo características antropológicas e culturais, as quais poderão gerar impacto no resultado final ao compararmos o respectivo escore entre uma população sadia e outra população referência com EM.

Nossos resultados demonstraram que no 25-FW o escore dos homens é significantemente maior se comparado com as mulheres, resultados estes semeIhantes aos encontrados na população alemã ${ }^{17}$.

No 9-HTP encontramos um escore significativamente melhor nas mulheres comparadas com os h omens, e um decréscimo no desempenho com o aumento da faixa etária, o que está de acordo com a observação de outros autores ${ }^{18-20}$.
Com relação ao PASAT, não observamos diferenças significantes no teste de 3 segundos, porém no teste de 2 segundos observamos melhor desempenho dos indivíduos de sexo masculino e naqueles com maior escolaridade, embora autores atribuam a correlação deste teste ao QI dos pacientes ${ }^{21}$ é bem demonstrada a interferência da escolaridade no desempenho final ${ }^{17}$.

A determinação dos pacientes analisados individualmente que tenham apresentado alteração significativa do escore-Z é polêmica, pois devemos considerar as características utilizadas na população referência antes de determinar o valor do cut-off para estabelecer mudanças estatisticamente significativas, motivo pelo qual não pudemos determinar este valor neste estudo. Estudos visando estabelecer o desvio padrão necessário para determinar as variações no escore da MSFC encontram-se em andamento 17,22.

A utilização da MSFC com esta população referência nos diversos centros de pesquisa em EM nos permitirá o melhor conhecimento da MSFC no portadores de EM no Brasil, uma vez que o teste já se mostrou significativo no nosso meio ${ }^{23}$.

\section{REFERÊNCIAS}

1. Rudick R, Antel J, Confraveux C et al. Clinical outcomes assessment in multiple sclerosis. Ann Neurol 1996;40:469-479.

2. Cohen JA, Fischer JS, Bolibrush DM et al. Intrarater and interrater reliability of MS functional composite outcome measure. Am Acad Neurol 2000;54:802.

3. Fischer JS, Rudick RA, Cutter GR et al. The MultipleSclerosis Functional Composite measure (MSFC): an integrated approach to MS clinical outcome assessment. Multiple Sclerosis 1999;5:244-250. 
4. Fischer JS, Jak AJ, Kniker JE et al. Administration and Scoring Manual for the MultipleSclerosis Functional composite Measure (MSFC). National Multiple Sclerosis Society. Canada. 1-40.

5. Kurtzke JF. Rating neurologic impairment in multiple sclerosis: an expanded disability status scale (EDSS). Neurology 1983;33:1444-1452.

6. Jacobs LD, Cookfair DL, Rudick RA et al. Intramuscular interferon beta 1 a for disease prog ression in relapsing multiple sclerosis. Ann Neurol 1996;39:285-294.

7. PRISMS Study Group. Randomized double-blind placebo-controlled study of interferon [beta]-1a in relapsing/remitting multiple sclerosis. Lancet 1998;352:1498-1504.

8. European Study Group on interferon [beta]-1b in secondary prog ressive MS. Placebo-controlled multicenter randomized trial of interferon [beta]-1b in treatment of secondary progressive multiple sclerosis. Lancet 1998;352:1491-1497.

9. Johnsons KP, Brooks BR, Cohen JA et al. Copolymer 1 reduces the relapse rate and improves disability in relapsing-renitting multiple sclerosis: results of a phase III multicenter, double-blind, placebo-controlled trial. Neurology 1995;45:1268-1276.

10. FelipeE, Mendes MF, Moreira MA et al. Análise comparativa entre duas escalas de avaliação clínica na esclerose múltipla: revisão de 302 casos. Arq Neuropsiquiatr 2000;58:300-303.

11. Amato MP, Fratiglioni L, Groppi C et al. Interrater reliability in assessing functional systems and disability on the Kurtzke scale in multiple sclerosis. Arch Neurol 1988;45:746-748.

12. Noseworthy JH, Vandervoort MK, Wong CJ, Ebers GC, the Canadian Cooperative MS Study Group. Interrater variability with the Expanded Disability Status Scale (EDSS) and Functional Systems (FS) in a multiple sclerosis clinical trial. Neurology 1990;40:971-975.

13. Sipe JC, Knobler RL, Braheny SL, et al. A neurologic rating scale (NRS) for use in multiple sclerosis. Neurology 1984;34:1368-1372.
14. Cook SD, Devereux C, Troiano R, et al. Effect of total lymphoid irradiation in chronic progressive multiple sclerosis. Lancet 1986;1:1405-1409.

15. Rudick RA, Antel J, Confavreux C, et al. Recommendations from the National Multiple Sclerosis Society Clinical Outcomes Assessment Task Force. Ann Neurol 1997;42:379-382.

16. Hobart J, Kalkares N, Barkhof F, et al . Outcome measures for multiple sclerosis clinical trials: relative measurement precision of the Expanded Disability Status Scale and MultipleSclerosis Funcional Composite. Multiple Sclerosis 2004;10:41-46.

17. Uitdehaag BMJ, Adèr HJ, Roosma TJA, et al. Multiplesclerosis functional composite: impact of reference population and interpretation of changes. Multiple Sclerosis 2002;8:366-371.

18. Kellor M, Frost J, Silberberg N et al. Hand strength and dexterity. Am J Occup Ther 1971;25:77-83.

19. Mathoiwetz V, Weber K, Kashman N et al. Adult normas for the Nine Hole Peg Test of finger dexterity. Occup Ther J Res 1985;5:24-38.

20. Mendes MF, Tilbery CP, Balsimelli S, et al. Teste de destreza manual da caixa e blocos em indivíduos normais e em pacientes com esclerose múltipla. Arq Neuropsiquiatr 2001;59:889-894.

21. Brittain JL, La Marche JA, Reeder KP et al. Effects of age and IQ on Paced Auditory Serial Addition Task (PASAT) performance. Clin Neuropsychol 1991;5:163-172.

22. Schwid SR, Goodman AD, ApatoffBR, et al. Quantitative measures are more sensitive to worsening neurological function in a prospective multiple sclerosis study. Am Neurol Assoc 1998;44:483.

23. Haase VG, Lima EP, Lacerda SS et al. Desenvolvimento da versão brasileira da Multiple Sclerosis Functional Composite Measure (MSFC). Arq Neuropsiquiatr 2004;62:363-370. 